\title{
Implementation of Embedded Agile Methodology
}

\author{
K.Jayashree \\ Research Scholar \\ Department of CSA, \\ SCSVMV University \\ Enathu, Kanchipuram
}

\author{
S.Babu \\ Asst.Professor \\ Department of CSA, \\ SCSVMV University \\ Enathur, Kanchipuram
}

\begin{abstract}
The main objective of Research is to implement embedded agile methodology using microcontroller. In earlier years agile methods have seen, individually, earn a worldwide following. The agile methods are used to indicate an advance in good practices of software development life cycle, but the advantages of agile methods changes across the different regions of software development. Embedded systems is an best illustration of a software development regime in which implementation of agile methods can be challenging, and the pros of using agile methods may not be as marked as in other systems of software development. This paper explains how the different aspects of embedded software development affect the usage of agile methods to field of embedded. Developers ,stakeholder,business and end user should see developed schedule performance and product quality. Developers should get capablity of regular feedback of iteractive development Tdd and spend a lot less time finding errors. Stakeholder, Business should see imporved certain and visible fact based of management data.Even though the whole result of using agile methods in embedded system software development is an improving efficiency, cautious method is needed to get the potential benefits.
\end{abstract}

\section{Keywords}

Sofware life cycle, Extreme Concepts, Iterative approach ,Microcontroller.

\section{INTRODUCTION}

The process of creation, installing, and regarding software has many fairly different stages: system requirements, system structure / design, component requirements , design, programming , automated testing, integration, and maintenance. Agile methods says that up-front design should be reduced. This is a best practice to study with volatile specifications and individual issues. Though the higher requirements for embedded systems are generally important more cut, dried, and frozen than specifications in the general software region. However embedded systems software development methods also requires to purpose for change, the method of answering to variation should be depend upon of change that is used to happen. Agile development methodologies have been provided to mention the problem of delivering high resolution software on time with constant and fast varying requirements and business place. Agile development practices are featured by elaborate programming practice, indepth communication between key stakeholders, low and bending teams and fast iterative cycles. It indicates an address to agile development and providing a brief introduction to the different agile methodologies, their features and key characteristics.

\subsection{Research Question}

The question of the focused research is "How to implement the agile software development methodology in hardware?".

\subsection{Objectives of thesis}

Agile software development is an looping process.we can uses this method for continuous changeing requirement or even it is very encouraged continuous iteration keep the system active to change and it also create close communiation between the user and developer and quickly responsive to the changes, the developer ensures that the growing and the ensuring system correctly accounts for the changes, test driven system make sure that only minimum software is used that it is develop correctly and that it remain exactly throughout the modifying changes.

\subsection{Scope of the research}

- To get good performance, the machine code must be well matched to the particular chip architecture.

- To have good clarity, the source code must be easily understandable by human reviewers. In theory, a compiler should be able to translate good-clarity source code into good-performance machine code, but in practice this translation is too complex to be completely feasible.

\section{LITERATURE REVIEW}

Agile methodology produces a lot of gains to managements who bended agility as their development process, many other managements competed tasks in providing agile on the member level or on the organization level. This implementation paper is a planned review that faces challenges when generating agile methods, still this paper fins out solutions to the challenges that were find out. Agile is improvingly get the ordered developing method in the software industry. A lot of managements are move towards agility in one way or another due to the requirement for fast delivery but at the same time provides with fast changing needs. While many of these managements have success in this to great extent, many others saw obstacles in their stages to vary from the old traditional methods to the modern agile The planned literature review is a new research method

,but it is considered as one of the methodologies of the Evidence-Based Software Engineering (EBSE) , and it is good more researchers in the field of software engineering .Generally the framework for the evidence-based software engineering (EBSE) was extracted from the working based practice in the medical and other research standards, and it can be mentioned as a "secondary" study that is depend on primary studies that were previously established. The net product of planned reviewing the recent research review on the use of agile methods and lean software development in 
global software engineering (GSE). The first purpose is to mention on which situations they have been applied effectively. Some general terms related to agile methods (e.g. scrum, extreme programming) were treated in arranging the search strings, along with a number of another ideas for GSE such as off shoring, outsourcing, and virtual teams.

\subsection{Software life cycle}

Various software development methodologies mention the steps differently. Some concentrate on or reject certain steps. Some develop continuously through the steps, but others permit for collapsing steps or cyclic repetition across steps. But, each step must be indicated at least in providing in any software development methodology .There are various formulas that can be used to find the "quality" of software. Many code quality procedures are related, but some are anticorrelated. In general, performance side such as cycles and memory usage are frequently anti-correlated with code quality features such as readability, testability, modularity, and maintainability. To provide best performance, the machine code must be well collided to the particular chip architecture. To produce good quality, the source coding must be easily acceptable by human reviewers. In general, a compiler should be able to change good-clarity source code into best performance machine code, but in practical this translation is too hard to be finished feasible. Basically, a compiler would produce highly tuned machine code from a normal language or simple graphical requirements, but compiler will not have enough artificial intelligence to do this within the unpredictable future.

\subsection{Reasons for Agile Development}

The following situations are used in the methods

- There is no Specifications in constant. Change in requirements is acted upon.

- Increased and repetitive method is used for modeling.

- Customers are involving in take part about modeling.

- Prioritization of requirements is being done by stakeholders and the team spirit on the highest requirement based on priority

- Every one in the team is an best participant and everyone's input to welcome.

- The context of the model is being find out as being more important than the styleor format.

\subsection{Two agile software development methodologies}

The most commonly used practices based on the agile philosophy are XP and Scrum. These differ in some features but share the repetitive method mentioned.

\subsubsection{XP}

$\mathrm{XP}$ stands for extreme programming. It focused on the development rather than managerial aspects of software projects. XP was produced so that managements would be free to bend all or part of the methodology.

\subsubsection{XP development}

$\mathrm{XP}$ practices begins with a open planning phase, followed by many iterations, each of which completes with user acceptance testing. When the product has enough characteristics to satisfy end user, the team end up the process of iteration and releases the software. Then they used to write "user stories" to explain the requirement of the software should satisfied. Those stories provide the team to evaluate the time and resources require to build the release and to define user acceptance tests. A user or a

representative is part of the XP team, so he or she can add all explanations to needs as the coding is being built. This permits needs to provide as both users and developers define how the product will look like. To produce a release system, the management breaks up the development requirements into repetitions. The final product provides each iteration plan, which makes the development for every repetition. At the last stage of an iteration, clients perform some tests against the stories of end user. If they find faults, fixing the bugs correction a step in the next. Iterative user acceptance testing, in general, can provide in release of the software. If users conclude that enough user stories have been given, the management can take to end the project before all of the normally planned user stories have been provided.

\subsubsection{XP rules and concept}

Development stages must involved changes into the development baseline at least once a day. This stage is also called continuous integration. The measurement of work done on all implementations is called Velocity. This significant formulas provides release planning and timing updates. All programs for a plan release is generated by teams working together at a single computer. XP provides that 2 programmers working together will satisfy user stories at the same speed as two programmers working alone, but with much higher quality.

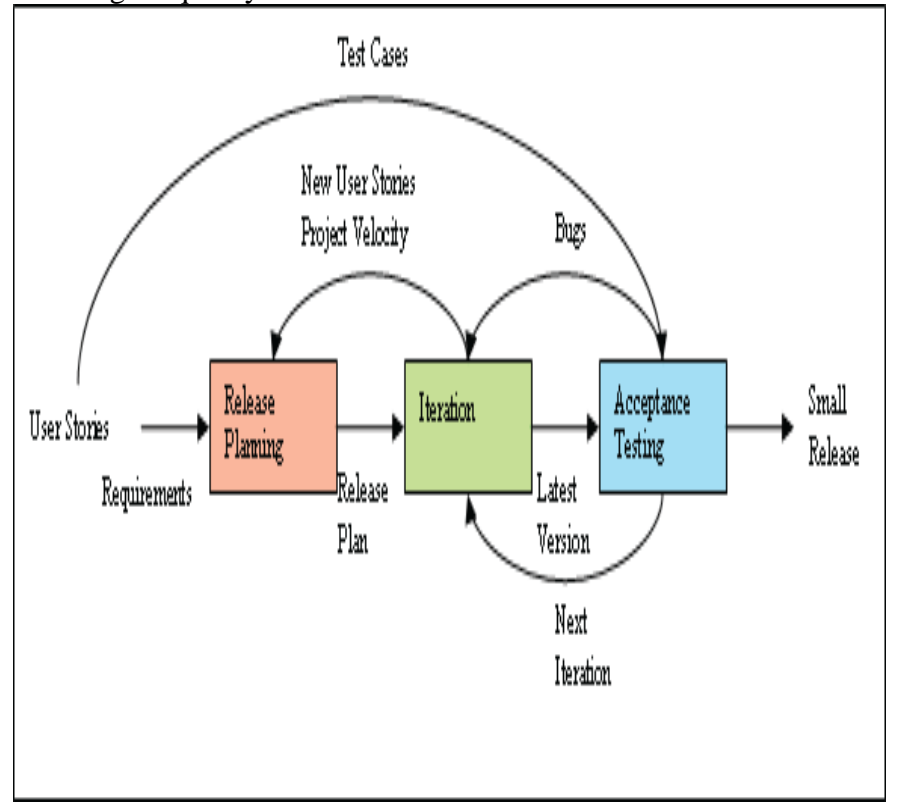

Fig 1. shows a simplified version of XP.

\section{RESEARCH METHODOLOGY}

The methodology of embedded agile is nothing but the implementing the agile software development method in embedded field. It consists of various process that gathering the specifications from the customers and the management team is divided into two categories such as developer and coder or programmer. The developer design the architecture for specifications design and the coder tested the design and release it after the satisfaction of end user or stakeholders requirements. This will be repeated if any feedback comes from the client in between. 


\subsection{The Agile Principles are}

- Priority is used to satisfy the customer through early and continuous provision of valuable software.

- When varying requirements, even late in development. Agile processes harness varies for the customer's complete merits.

- Completed working software often, from a couple of weeks to a couple of months, with a priority to the shorter timescale.

- Business team and developers must work together daily throughout the termination of project.

- The primary is best practice software.

- The process of increasing the amount of work which is not done-is significant is called as simplicity.

- The best structures, needs, and designs that produced from self-organizing teams.

- At repetitive intervals, the team says on how to increase more effective, then adjustment is required.

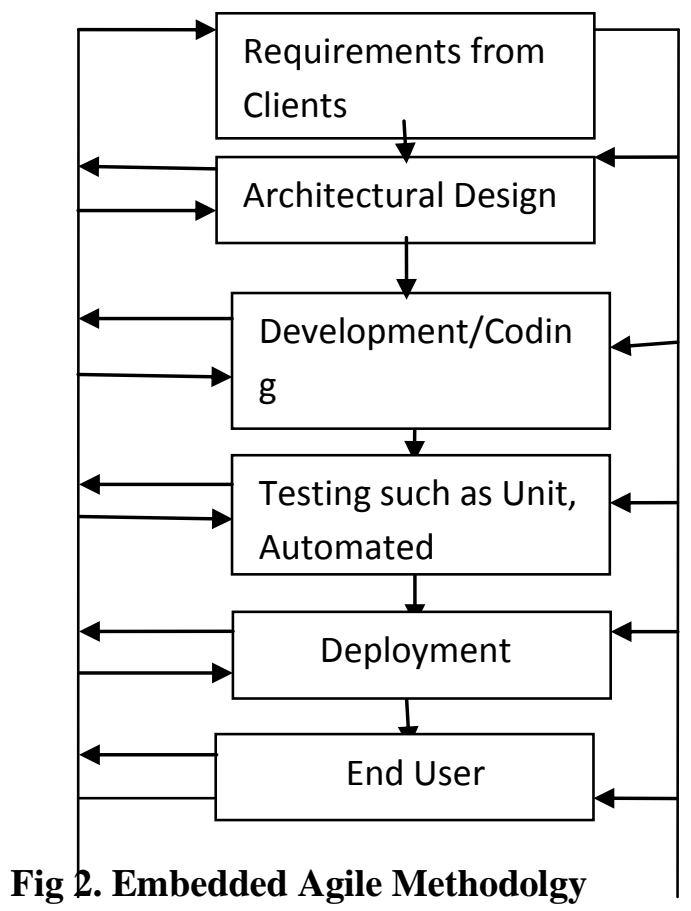

\subsection{Iterations}

Agile development is depends on iterative and incremental development .Iterative gives general feedback by dividing the task into iterations that are commonly one or two weeks in length. The output of each iteration is best practiced software. Each iteration is like a individual project wind up after regular amount of time, and produced some executable version of the product. In last years repetitions the software may run in a test environmentorprototype.

There are 2 main functions in agile development, the developer role, and the customer role. 2 groups of people commonly indicates each role. In a simple words, the end user defines and tests the product and the development gives the delivery product
Needs to be break up into into smaller individual units also called stories. They are the testable, estimateable and deliverable units. They are low enough so that all can be finished within a one iteration. When the developers finish a story the users get feedback on the needs by watching and touching what has been developed. In the early repetitions, first preference to hardware availability some of these stories are explained through tests and simulations; but the working are based on repeated code. In the method of waterfall situation, the time is spent up front on products that may never make the deliver product. In Agile development some tries is first started on the highest priority characteristics and capabilities, needs are elaborated in parallel with development and finally little time is gone. Hope it can be explained in detail level. The repetitive method to needs gathering, reduction of damage and development methods means that variations in user needs, work goals and hardware structure are more naturally occupied. Time to be used in market can be increased by deleting some unused software in the development process by satisfy the needs, software and hardware trains on three parallel stages.

\subsection{Automated Test}

The hard and some nature of software development means there are various opportunities to stop existing practiced software.

The automated test of agile method and acceptance tests makes repeated tests in a simple manner. The cheap in running all the unit tests whenever any variation is made to the source code. This is complex to do, particularly when you are best learning, but the enough time is saved will outweigh the time you generally would waste manually testing and debugging. Unit tests support the thing that new characteristics should not affect existing features. These tests are a very good investment. They make testing a repeatable process, that can be run with every variations.

\subsection{Advantages of Agile Development}

- Less duration in development cycles

- More stable of deliverables occurs.

- Increased usage of resources

- The quality of final product due to the involvement of the customer is better.

- The method needs good concentrated and skilled individuals which would not always be existent.

\section{RESULTS AND DISCUSSION}

The results of embedded agile methodology describes the concept difference applied in embedded field. The requirements are gathered and the procedure can be deployed with certain procedures through waterfall method. Now, some improvements can be developed through agile methodology to satisfy the end user or stakeholders. 


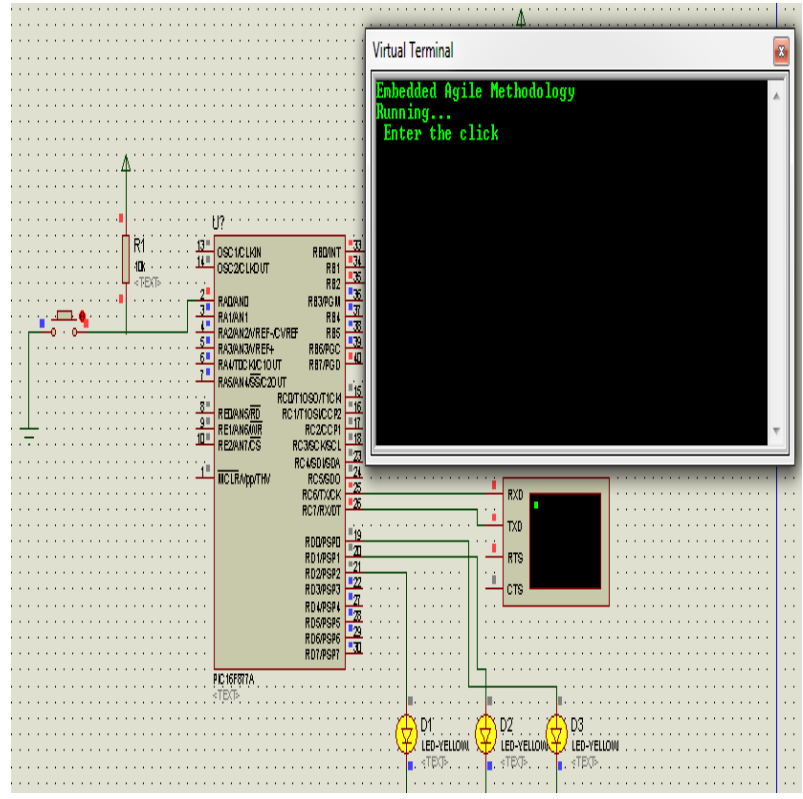

Figure 3.Simulated output for task-1

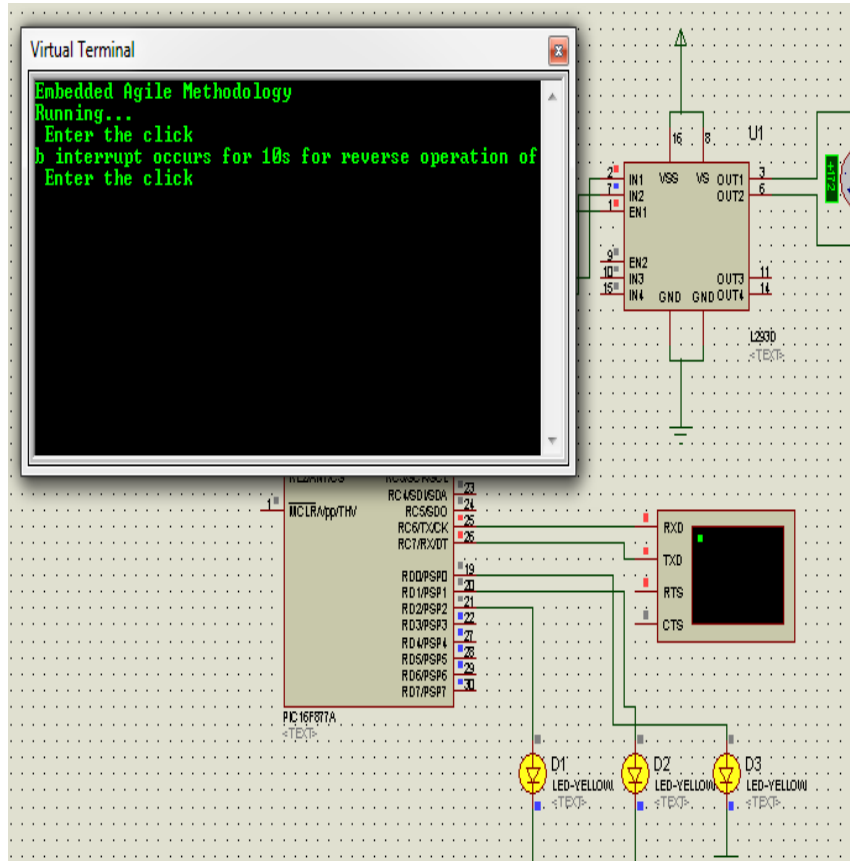

Figure 4.Simulated Output for Interrupted Task

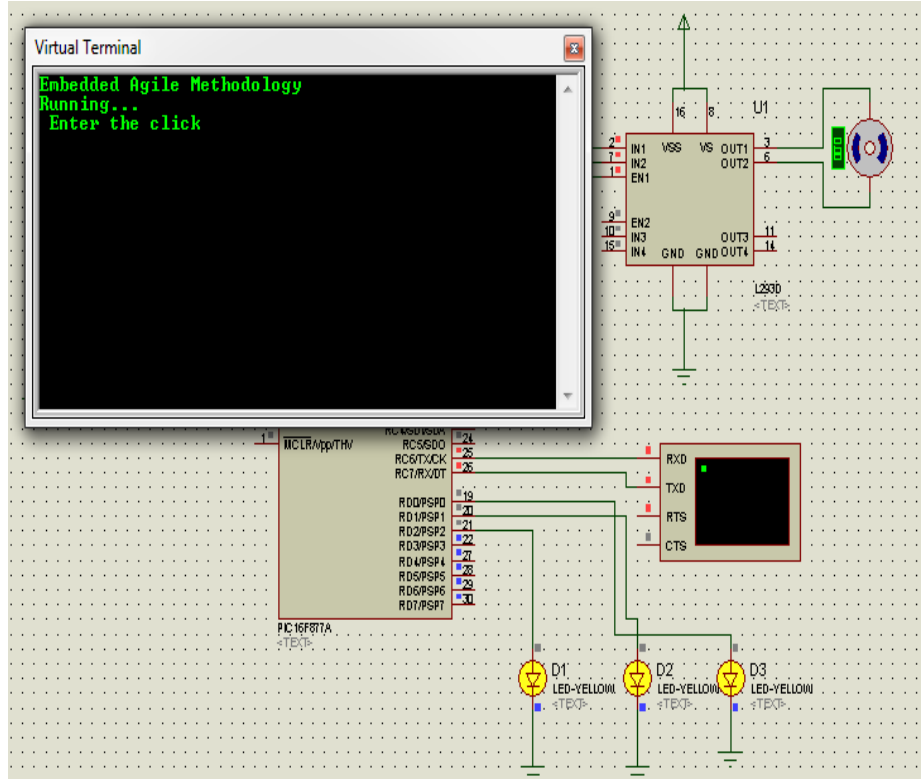

Figure 5. Iterative Approach for Embedded Method

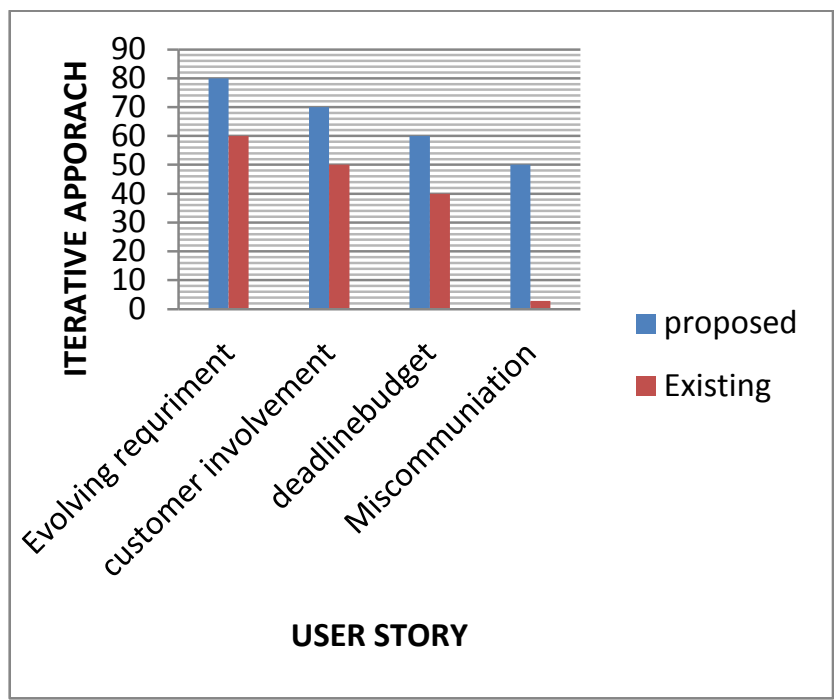

Figure 6. comparison between existing and proposed Embedded agile metholodogy

\section{CONCLUSION}

Thus, it have been concluded that embedded agile methodology is implemented with the iterative procedure. The requirements are collected from the stakeholders and some task is implementing using the developer and then code is tested for end user. While satisfying the task of operating dc motor some interrupt will occur and motor will be operated in reverse direction iterative approach is done till the satisfaction of end user.

This implementation explains the characteristics of generating Agile approaches to the development of hardware for the processor family. Embedded development tasks are simple distinct from object-oriented and pure software customers ,but they saw many of the same difficulties that Agile software development practices address. Many different difficulties are explained, that includes team workers mentioned domain concept, technical knowledge and 
thoughts toward variations, and the aspect on which the hardware plays in hardware design. The Agile methods to be well-suitable for our concept, that the truth that most Agile methodologists come from very different methods.

Simple as they are, those particular needs have to be suitable in hours or days rather than weeks or months. Low quality or late update into the bus hardware would affect hard cost and overhead. The software engineering methods were considered as not sufficient and needed to be less .

Agile methods provides guidance for quality, flexibility and high speed need to be recycled. Though, we did not bend any full agile method, but requires single agile practices to our "process development toolbox. The number of classical process to increase activities (such as more planned documentation and measurement) and joined them with agile elements (e.g. Test First Process). This mixing seemed to faster and acceptance of agile ideas that may used for us to reduce the ice for a cautious extension of agile method. improvement.

\section{REFERENCES}

[1] Charles E.Mathews Fifth Generation Systems Ltd,2011.Agile Practices in Embedded Systems Markham,On,Canada Publication year 2011 view colleagous of architectureal designfor programm development IBM systems.

[2] MattFletcher.William

Bereza,Atomic Object,LLC,2010.Evolving into Embedded development

[3] Carl Ericksson,RaplhPlamer,Dvid Crosbyl,Michael Marsiglial,Micah Alles,2012 Make Haste,Not Waste:Automated system Testing XP Agile Universe New Oreceans,LA,USA July2003

[4] Sabah Nouri Mohammed Hussian,2009.Agile Method Implementation-Gupea University Technology, Department of Computer Sci Engineering.

[5] Samireh Jalali,Gobal Software Engineering and Agile Practise Systematic Review Journal of Software Maintenance and Evolution Research $20105^{\text {th }}$ International Conference.
[6] Claes Wohlin,"Agile Practices in Gobal Software Engineering and Extreme Programming" Processing of the $13^{\text {th }}$ international $\mathrm{Xp}$ conference,Malmo,Sweden Springer,ISBN 978-3-642-30349-4,2008

[7] Mike Karlesky,and Greg Williams, Embedded Development ,The Social Nture of Agile Teams Publication Year 2010

[8] Tsun,Chow,Dac-Buu Cao "A Survey Study of Critical Success Factor in AGILE Software Projects in School of Business and Technology, capella University,Minneapolis publication year 2008 ,pp 961971

[9] T. Dybå, T. Dingsøyr, "Empirical Studies of Agile Software Development: a Systematic Review", Journal of Information and Software Technology 50 (2008), 2008, pp. 833-859.

[10] P. Abrahamsson, J. Warsta, M.T. Siponen, J. Ronkainen, "New Directions on Agile Methods: a Comparative Analysis", Proceedings of the 25th International Conference on Software Engineering, ACM Press, 2003, pp. 244-254.

[11] B. Boehm, R. Turner, "Management Challenges to Implement Agile Processes in Traditional Development Organizations", IEEE Software (22)5, 2005, pp. 30-39.

[12] H. Sharp, H. Robinson, "An Ethnographic Study of XP Practice", Journal of Empirical Software Engineering 9(4), 2004, pp. 353-375.

[13] R. Wieringa, N.A.M. Maiden, N.R. Mead, C. Rolland, "Requirements Engineering Paper Classification and Evaluation Criteria: a Proposal and a Discussion", Journal of Requir. Eng. 11(1), 2006, pp. 102-107.

[14] P.J. Ågerfalk, B. Fitzgerald, H. Holmström, B. Lings, B. Lundell, E. Ó Conchúir, "A Framework for Considering Opportunities and Threats in Distributed Software Development", Proceedings of International Workshop on Distributed Software Development, Austrian Computer Society, 2005, pp. 47-61.

[15] Jussi.ronkainen,Pekka.Abrahamsson,p.(2003)Software development under stringent hardware constraints:Do agile have a chance?InXP2003,Genova,Italy 